\title{
Three Dimensional Microanatomy of the Ophthalmic Artery: Spontaneous Intracranial-Extracranial Anastomosis Site within the Orbital Cavity
}

\author{
Enis KURUOGLU, Cengiz COKLUK, Abdullah Hilmi MARANGOZ, Kerameddin AYDIN \\ Ondokuzmayis University, School of Medicine, Department of Neurosurgery, Samsun, Turkey
}

\section{ABSTRACT}

AIM: The aim was to evaluate three-dimensional structure of the ophthalmic artery (OphA).

MATERIAL and METHODS: The raw data of the Three-dimensional Computerized Tomography Angiography (3D-CTA) were transferred to computer and recorded in a software program.

RESULTS: The study group consisted of 45 ( 24 female and 21 male) patients. The mean age of the patients was $56 \pm 9.5$ years. It was found that the entry point of the OphA to the orbital cavity was inferior to the optic foramen in $29(64.44 \%)$ of the cases. The length of the postcanalicular-prelacrimal was $0.54 \pm 0.13$ centimeters at the right side and $0.51 \pm 0.13$ centimeters at the left side. The distance of the postlacrimal-presupraorbital segment was $0.43 \pm 0.11$ centimeters at the right and left sides. The location of the anastomosing site was $0.97 \pm 0.42$ centimeters from the supraorbital notch.

CONCLUSION: The results of this study showed us that this technique could be used in the evaluation of the three-dimensional microsurgical anatomy of the OphA and its critical extracranial-intracranial anastomosing site.

KEYWORDS: Ophthalmic artery, Volume rendering, Microsurgical anatomy, Three-dimensional images, Computerized tomography

\section{INTRODUCTION}

The ophthalmic artery (OphA) is the first branch of the internal carotid artery distal to the cavernous sinus. The OphA supplies the structures within the orbit as well as some structures in the nose, face and meninges. The OphA arises from the internal carotid along the medial side of the anterior clinoid process and runs anteriorly passing through the optic canal inferolaterally to the optic nerve. The central retinal artery (CRA) is the first, and one of the smaller branches of the OphA. The next branch of the OphA is the lacrimal artery. It is one of the largest branches within the orbital cavity.

The supraorbital artery is the second major branch of the OphA. Around the supraorbital notch, the OphA has some critical spontaneous intracranial-extracranial anastomoses with the terminal branches of the facial artery.
The volume rendering technique may be used in the threedimensional evaluation of some anatomical structures such as OphA. The volume rendering technique is a group of modalities in the conversion of two-dimensional images to threedimensional images $(2,5,12)$. The two-dimensional images acquired by computerized tomography (CT) and magnetic resonance imaging is used to create the volume rendered images $(2,4,5)$. Three-dimensional (3D) reconstructed images accurately reflect the real measurement in patients who have undergone multislice helical CT examination (15). In this study, we used the OsiriX software program for volume rendering technique to create three-dimensional ophthalmic artery images. The results of this study and the feasibility of this technique are discussed under the light of published medical literature. 


\section{MATERIAL and METHODS}

Any additional radiological examination and providing any drug were performed to the patients for this study. The patient population included the cases that were brought to our neurosurgery department because of subarachnoid hemorrhage, and where a decision was made for further 3D-CTA investigation for cerebral aneurysm evaluation. The raw data of the 3D-CTA were transferred and recorded to a computational software database. The purpose of performing 3D-CTA was only the examination of the intracranial vascular pathology after the insult of subarachnoid hemorrhage. Some part of these raw data were used for examination of the three dimensional anatomy of the OphA and its spontaneous extracranial-intracranial anastomoses with the branches of the facial artery. Any additional procedure was done any of the patients. Imaging data were stored in the digital imaging and communications in medicine (DICOM) format and subsequently analyzed with imaging software to convert into the three-dimensional volume rendered neurovascular images. The computer program created the three-dimensional OphA images using these transferred raw data with the volume rendering technique. Three-dimensional images of the OphA were evaluated in terms of general shape, emerging point from the optic foramen, length, and the site of the spontaneous intracranial-extracranial anastomoses with the branches of the facial artery.

\section{RESULTS}

The study group consisted of 45 (24 female and 21 male) patients. The mean age of the patients was $56 \pm 95$ years. Any additional radiological procedure and/or drug were performed to any patient for this study. The purpose of the radiological examination of the patients was only to diagnose and evaluate their own disorder. The radiological images from this examination were retrospectively transferred to a computer. Three Dimensional OphA images were created with 3D-Volume Rendering Technique using the OsiriX MD software program.

The entry points of the OphAs through the optic foramen was evaluated by using three-dimensional images (Figure 1). The emerging site of the OphA from the medial site of the internal carotid artery and its entry to the optic canal is shown in Figure 1. The surface of the optic foramen to the ophthalmic cavity was divided into four-quarter sections by vertical and axial planes passing at the central point of the foramen (Figures 2, 3). The inferior half of the foramen included three different parts as inferior-lateral, middle-inferior, and inferior-medial (Figure 3). The dividing of the optic foramen is shown in Figure 3. The OphA exited from the foramen through the middle inferior part in 29 (64\%) of the cases (Figure 4). The inferior-lateral part was found in $9(20 \%)$ of the cases. In five (11\%) of the cases, the OphA passed to the ophthalmic cavity through the inferior medial side of the optic foramen. In the remaining two $(5 \%)$ cases, the existing point was found to be the lateral side of the ophthalmic foraminal opening.

The OphA was divided into four segments as canalicular (O1), postcanalicular-prelacrimal (O2), Postlacrimal-presupraorbital

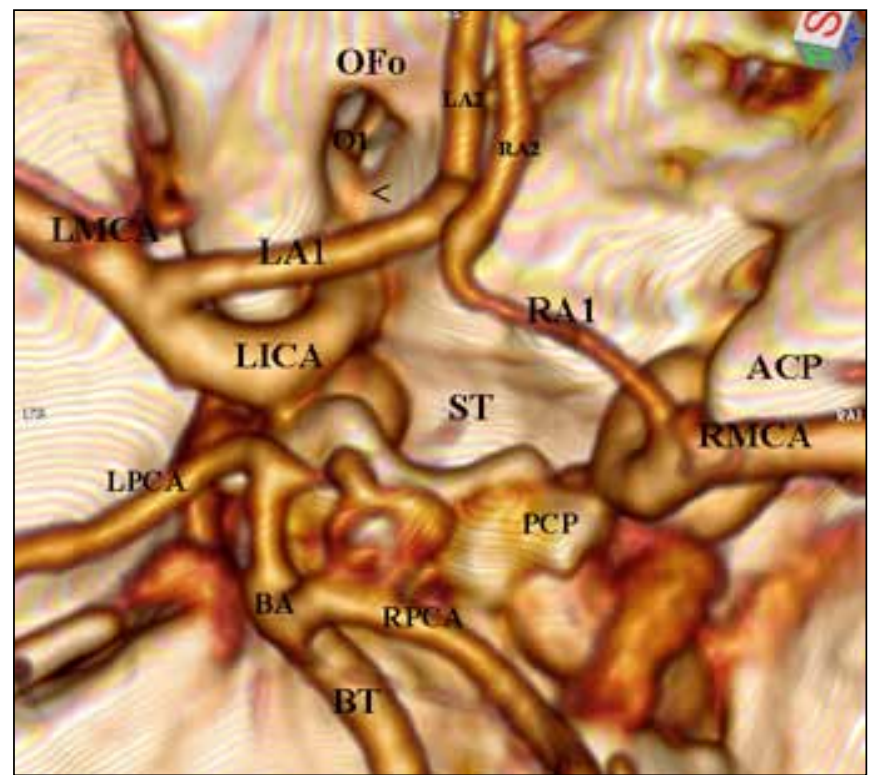

Figure 1: The emerging of the OphA along the medial site of the internal carotid artery and its entrance to the optic canal, and the canalicular (O1) segment are shown in this figure (OFo: optic foramen; LA2: left anterior cerebral artery A2 segment; RA2: right anterior cerebral artery A2 segment; 01: canalicular segment of the OphA; RA1: right anterior cerebral artery A1 segment; LA2: left anterior cerebral artery A1 segment; LMCA: left middle cerebral artery; RMCA: right middle cerebral artery; LICA: left internal carotid artery; ACP: anterior clinoid process; ST: sella turcica, PCP: posterior clinoid process; LPCA: left posterior cerebral artery; RPCA: right posterior cerebral artery; BA: basilar apex; BT: basilar artery trunk; arrow shows the emerging point of the OphA along the medial site of the internal carotid artery).

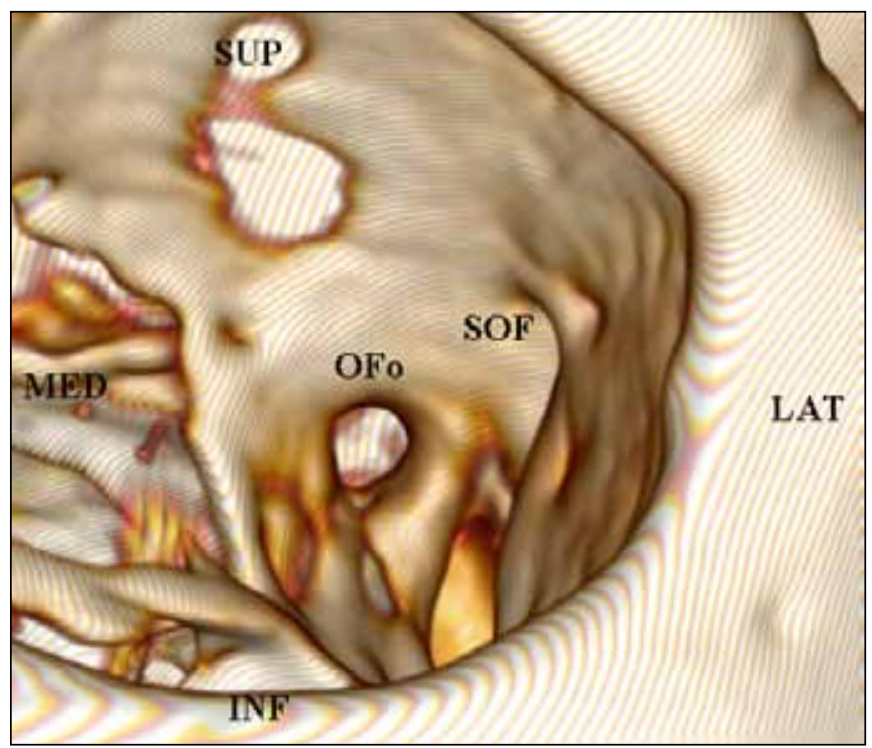

Figure 2: The location of the optic foramen (SUP: superior; SOF: superior orbital fissure; LAT: lateral; MED: medial, INF: inferior). 
(O3), and postsupraorbital or terminal (O4) (Figure 5). The segmentation of the OphA is shown in Figures 5 and 6 . The emerging point of the supraorbital artery is the border of the postsupraorbital or terminal segment. The length of the

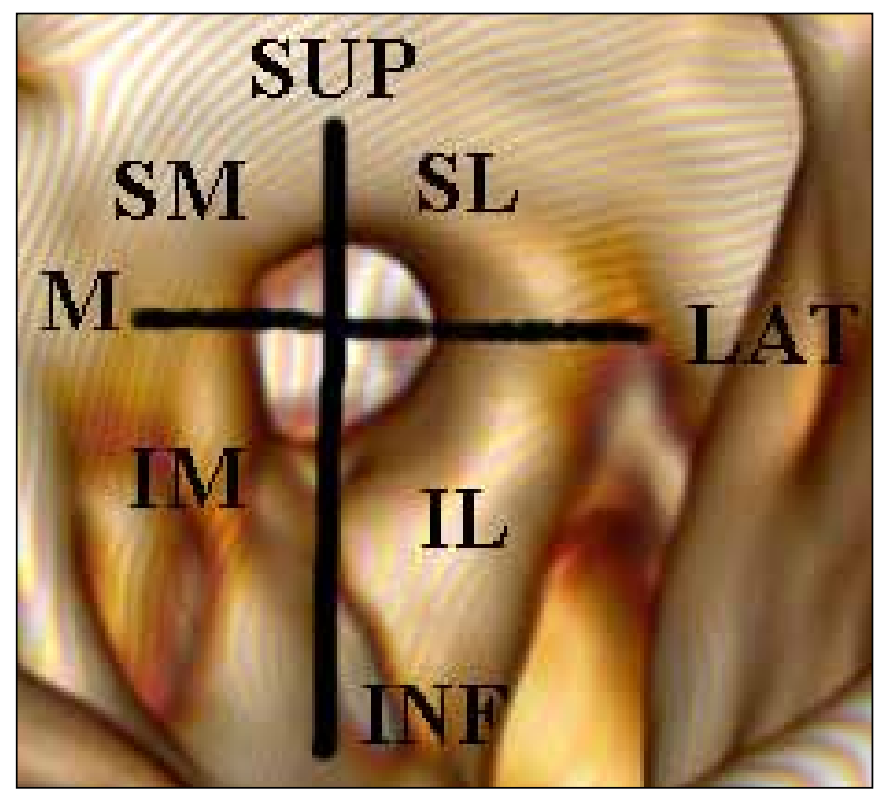

Figure 3: The dividing of the optic foramen with two imaginary lines (SUP: superior; LAT: lateral; M: medial; INF: inferior; SM: superior-medial, SL: superior lateral; IM: inferior-medial; IL: inferior lateral).

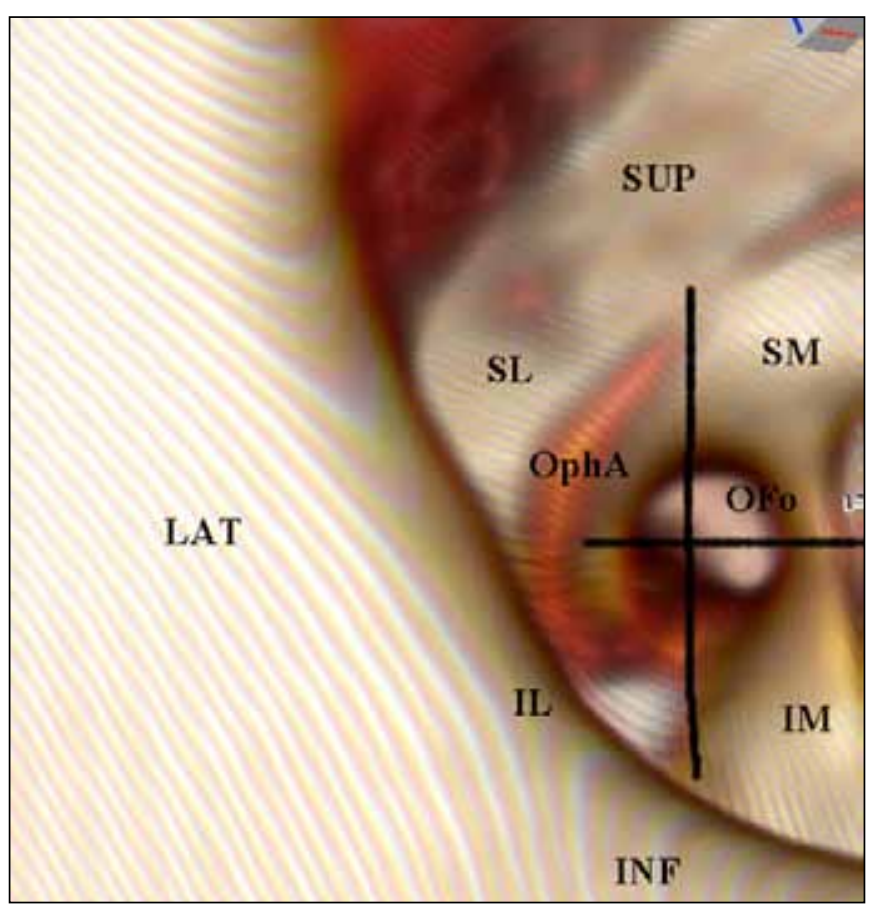

Figure 4: The exit of the OphA from the optic foramen (SUP: superior; LAT: lateral; INF: inferior; SM: superior-medial, SL: superior lateral; IM: inferior-medial; IL: inferior lateral; OphA: ophthalmic artery; OFo: optic foramen). prelacrimal segment was $0.54 \pm 0.13$ centimeters at the right side and $0.51 \pm 0.13$ centimeters at the left side. The distance of the postlacrimal-presupraorbital segment was $0.43 \pm 0.11$ centimeters at the right and left sides. The determination and evaluation of the postsupraorbital or terminal segment was not optimally done using this technique in the majority of the cases because of the calibration of the artery and imagination difficulties.

The site of the spontaneous intracranial-extracranial anastomosing with the angular artery was also evaluated in these images. The quality of the images in the evaluating of the anastomosis was quite enough in the majority of the cases. The location of the anastomosing site was found to be $0.97 \pm 0.42$ centimeters inferior-medial from the supraorbital notch.

\section{- DISCUSSION}

Three-dimensional anatomical details of the OphA can be imagined using the volume-rendering technique. Gross microscopic and microsurgical anatomy of this vascular structure may be evaluated using these images. The OphA is the first branch of the internal carotid artery after passing through the cavernous sinus. The OphA supply the structures within the orbit as well as some structures in the nose, face and meninges. The OphA arises from the internal carotid along the medial side and runs anteriorly passing through the optic canal inferolaterally to the optic nerve. The CRA is the first, and one of the smaller branches of the OphA. The next branch

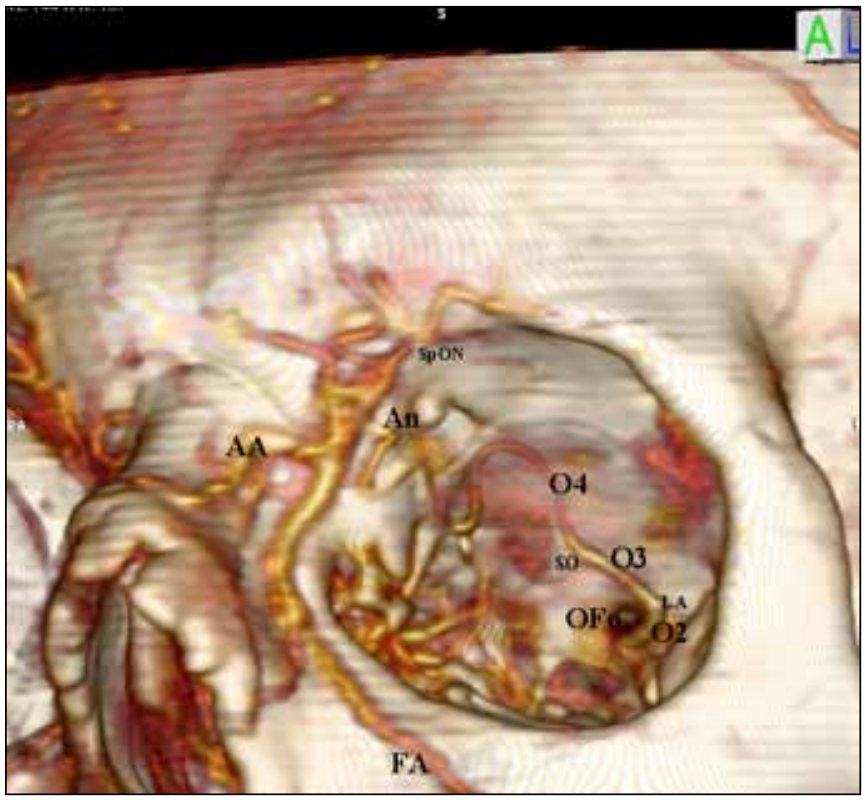

Figure 5: The segmentation of the ophthalmic artery and its anastomosis with angular artery (SpON: Supraorbital notch; An: anastomosis; AA: angular artery; FA: facial artery; 04: postsupraorbital segment; SO: emerging site of the supraorbital artery; 03: postlacrimal-presupraorbital segment; LA: emerging site of the lacrimal artery; OFo: optic foramen; 02: prelacrimal segment of the ophthalmic artery). 


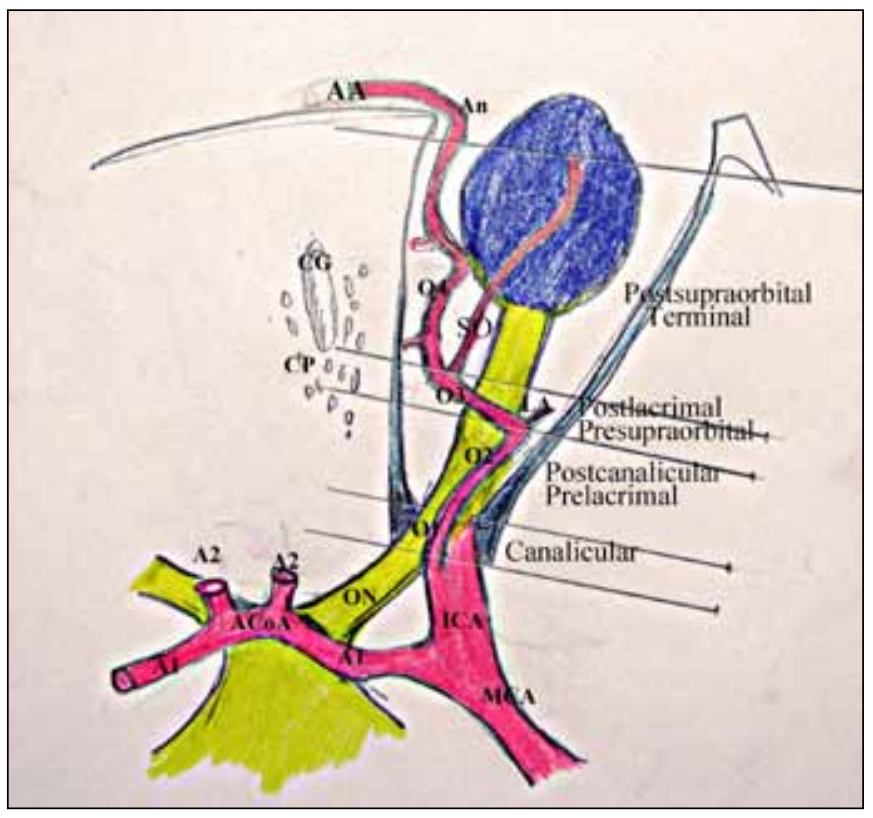

Figure 6: The segments of the ophthalmic artery (AA: angular artery; An: anastomosis; CG: crista galli; CP: cribriform plate; O4: postsupraorbital or terminal segment; SO: supraorbital artery; 03: postlacrimal-presupraorbital segment; LA: lacrimal artery; 02: postcanalicular-prelacrimal segment; 01: canalicular segment; ICA: internal carotid artery; MCA: middle cerebral artery; ON: optic nerve; A1: anterior cerebral artery A1 segment; ACoA: anterior communicating artery; A2: anterior cerebral artery A2 segment).

of the OphA is the lacrimal artery. It is the one of the largest branches within the orbital cavity. The supraorbital artery is the second major branch of this artery.

Digital Subtraction Angiography (DSA) is still the most sensitive diagnostic procedure in the evaluation of intracranial and extracranial vascular lesions such as aneurysms and arteriovenous malformations (12). At the same time, digital subtraction angiography is expensive, invasive, and brings an associated $1.5 \%$ to $2.0 \%$ risk of significant morbidity and mortality (13). Computerized tomographic angiography with its three dimensional advantage that can depict the arterial lumen and wall is a fast and inexpensive tool and widely available technique for intracranial aneurysm detection. Intracranial aneurysms can be easily diagnosed with a performance that can be comparable to that of DSA (1). In the published literature, the diagnostic sensitivity of computerized tomographic angiography was reported between the range of $70 \%$ and $96 \%$ depending on the size and location of the pathology $(7,10,11,14)$. In addition to that; CT angiography data sets can be used to select to treat aneurysm and thus avoid only performing diagnostic DSA (1). Furthermore, CT angiography has high utility and some abnormalities determined by CT angiography have a strong prognostic value (9). Nevertheless, it still has limited sensitivity in detecting aneurysms less than $3 \mathrm{~mm}$ despite the technical advances of CT angiography mentioned above (1).
The volume rendering technique was first developed based on research at the Mayo Clinic in the 1970s (2). Following advances in image processing hardware and integration of new data manipulation techniques were developed by University of North Carolina and Pixar (4, 8). After this date, some important progress was achieved in parallel with hardware and software advancement in computer technology as well as image processing technology in neurological radiology. According to literature records, it may be concluded that cadaveric and CT angiographic measurements are similar and overlap with each other $(3,6)$. This may provide considerable intraoperative safety in surgical approaches related to anatomical structures such as OphA. When preoperative CT angiography is performed, the best surgical approach can be determined and potential complications can be avoided. OsiriX is software for radiological image processing. Using the software can provide three-dimensional images. The three-dimensional viewer provides modern rendering modes such as multiplanar reconstruction, surface rendering, volume rendering, and maximum intensity projection. In the present study, we used the OsiriX software to process DICOM images. This software may show the basal cerebral arteries and OphA together with the bone structure of the cranial base and orbital cavity.

The OphA enters the orbital cavity through the optic foramen. The entry points were evaluated by using three-dimensional volume rendered images (Figure 1). The optic foramen was clearly imagined in all cases (Figure 2). Before the evaluation, we divided the surface of the foramen facing the orbital cavity into four equal parts with two imaginary lines (vertical and axial lines) passing through the central point of the foramen (Figures 3, 4). The imaginary axial line divided the foramen into equal halves as superior and inferior parts. The inferior half of the foramen could be divided as inferior lateral, middleinferior and inferior medial parts. The exit site of the OphA was evaluated according to these parts. Generally the OphA left via the optic foramen to the orbital cavity through the middleinferior part of the foraminal opening as seen in $64 \%$ of the cases in this group (Figure 4). The second group was the inferior lateral group including $20 \%$ of the cases. The lateral exit was found to be very rare.

The segmentation of the OphA can be done according to related anatomical structures around and/or passing through the artery. The OphA was mainly divided as four parts as canalicular (O1), postcanalicular-prelacrimal (O2), postlacrimal-presupraorbital (O3) and postsupraorbital or terminal $(\mathrm{O} 4)$. The canalicular part is totally located inside the optic canal with the optic nerve. This part starts at the emerging point from the medial side of the internal carotid artery and ends at opening of the optic foramen to the intraorbital cavity. The second main part is located within the orbital cavity. It is the segment located between the intraorbital opening of the optic canal and emerging point of the lacrimal branch of the OphA. The length of the prelacrimal segment was found to be $0.54 \pm 0.13$ centimeters at the right side and $0.51 \pm 0.13$ centimeters at the left side. The postlacrimal segment is located between the lacrimal artery and supraorbital artery. The distance of the postlacrimal-presupraorbital segment was $0.43 \pm 0.11$ centimeters at the right and left sides. 
The location and calibration of the spontaneous intracranialextracranial critical anastomoses of the OphA with the branches of the facial artery can also be evaluated using three-dimensional volume rendered images in cases with subarachnoid hemorrhage. The results of the present study revealed that the location of the anastomosis site is located $0.97 \pm 0.42$ centimeters inferior and lateral from the supraorbital notch. The imagination and evaluation of this place is important especially in the cases with obstructive internal carotid artery disease proximal to the emerging point of the OphA. This location should be taken into account in cases with obstructive intracranial vascular diseases.

\section{CONCLUSION}

Three-dimensional images of the head including bone and vascular structures may provide some specific dynamic information. The microsurgical anatomy of the OphA could be examined using three-dimensional volume rendered images from contrast enhanced computerized tomographic examination in the cases with subarachnoid hemorrhage. Digital estimations of the segments may also be made using these images. The spontaneous intracranial-extracranial anastomosis of the OphA with the terminal branches of the facial artery can also be evaluated using this imaging modality.

\section{- REFERENCES}

1. Badry A, Elshafey R, Khalil M: Detection, characterization and endovascular therapy planning of intracranial aneurysms with 16-channel multidetector row CT angiography. The Egyptian Journal of Radiology and Nuclear Medicine 45:151-158, 2014

2. Calboun PS, Kuszyk BS, Heath DG, Carley JC, Fishman EK: Three-dimentional volume rendering of spiral CT data: Theory and Method. RadioGraphics 19(3): 745-763, 1999

3. Cengiz SL, Cicekcibasi A, Kiresi D, Kocaogullar Y, Cicek O, Baysefer A, Buyukmumcu M: Anatomic and radiologic analysis of the atlantal part of the vertebral artery. J Clin Neurosci 16(5):675678, 2009

4. Drebin RA, Carpenter L, Hanrahan P: Volume rendering. Comput Graph 22: 65-74, 1988
5. Hwang SB, Kwak HS, Han YM, Chung GH: Detection of intracranial aneurysms using three dimensional multidetector-row CT angiography: Is bone substraction necessary? European Journal of Radiology 79: e18-e23, 2011

6. Kahilogullari G, Ugur HC, Comert A, Tekdemir I, Kanpolat Y: The branching pattern of the middle cerebral artery: Is the intermediate trunk real or not? An anatomical study correlating with simple angiography. J Neurosurg 116(5):1024-1034, 2012

7. Karamessini MT, Kagadis GC, Petsas T, Karnabatidis D, Konstantinou D, Sakellaropoulos GC, Nikiforidis GC, Siablis D: CT angiography with three-dimensional techniques for the early diagnosis of intracranial aneurysms: Comparison with intraarterial DSA and the surgical findings. Eur J Radiol 49: 212-223, 2004

8. Levoy M: Display of surfaces from volume data. IEEE Comput Graph Applicat 8: 29-37, 1988

9. Schlett LC, Nance JW Jr, Schoepf UJ, O’Brien TX, Ebersberger $U$, Headden GF, Hoffmann U, Bamberg F: Differences in coronary artery disease by CT angiography between patients developing unstable angina pectoris vs. major adverse cardiac events. European Journal of Radiology 83:1113-1119, 2014

10. Schwartz RB, Tice HM, Hooten SM, Hsu L, Stieg PE: Evaluation of cerebral aneurysms with helical CT: Correlation with conventional angiography and MR angiography. Radiology 192: 717-722, 1994

11. Teksam M, McKinney A, Casey S, Asis M, Kieffer S, Truwit CL: Multi-section CT angiography for detection of cerebral aneurysms. AJNR Am J Neuroradiol 25: 1485-1492, 2004

12. Tomandi BF, Hammen T, Klotz E, Ditt H, Stemper B, Lell M: Bonesubstraction CT angiography for the evaluation of intracranial aneurysms. AJNR Am J Neuroradiol 27:55-59, 2006

13. Waugh JR, Sachara N: Arteriographic complications in the DSA era. Radiology 182: 243-246, 1992

14. White PM, Wardlaw JM, Easton V: Can noninvasive imaging accurately depict intracranial aneurysms? A systematic review. Radiology 217: 361-370, 2000

15. Zhilhai L, Qixue G, Baohong T, Jingyao L, Zhiyi C: Three dimensional reconstruction of optic canal-based on multislice helical CT: A comparison analysis with skull dissection of 40 postmortem cases. Anat Rec (Hoboken) 291(12):1662-1672,2008 